\title{
Erratum to: An Italian consensus for invasive candidiasis management (ITALIC)
}

\author{
L. Scudeller $\cdot$ C. Viscoli $\cdot$ F. Menichetti $\cdot$ \\ V. del Bono $\cdot$ F. Cristini $\cdot$ C. Tascini $\cdot$ \\ M. Bassetti $\cdot$ P. Viale
}

Published online: 8 January 2014

(c) Springer-Verlag Berlin Heidelberg 2013

\section{Erratum to: Infection \\ DOI 10.1007/s15010-013-0558-0}

In the original article, unfortunately the list of authors was incomplete.

The correct author list is as follows:

L. Scudeller · C. Viscoli · F. Menichetti · V. del Bono . F. Cristini - C. Tascini - M. Bassetti - P. Viale · the ITALIC group

The online version of the original article can be found under doi:10.1007/s15010-013-0558-0.

L. Scudeller $(\bowtie)$

Clinical Epidemiology Unit, Scientific Direction, IRCCS

Policlinico San Matteo Foundation, P.le Golgi 2,

27100 Pavia, Italy

e-mail: 1.scudeller@smatteo.pv.it

C. Viscoli · V. del Bono

Clinic of Infectious Diseases, IRCCS San Martino-IST,

University of Genua, Genoa, Italy

F. Menichetti $\cdot$ C. Tascini

Infectious Disease Department, Cisanello Hospital, Pisa, Italy

F. Cristini $\cdot$ P. Viale

Infectious Diseases Unit, Teaching Hospital Policlinico S.

Orsola-Malpighi Alma Mater Studiorum, University of Bologna,

Bologna, Italy

M. Bassetti

Infectious Diseases Division, Santa Maria della Misericordia

University Hospital, Udine, Italy
Members of the ITALIC group:

Chiara Adembri, Massimo Antonelli, Giacomo Borgonovo, Francesco Bruno, Ercole Concia, Francesco Giuseppe De Rosa, Vincenzo Emmi, Silvano Esposito, Roberto Fumagalli, Massimo Girardis, Paolo A. Grossi, Roberto Luzzati, Paolo Malacarne, Daniela Pasero, Paolo Pelosi, Nicola Petrosillo, Massimo Sartelli, Gabriele Sganga, Liana Signorini, Romano Tetamo, Mario Tumbarello, Mario Venditti. The ITALIC group members are listed with their complete affiliations at the end of the article. 